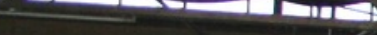

4.
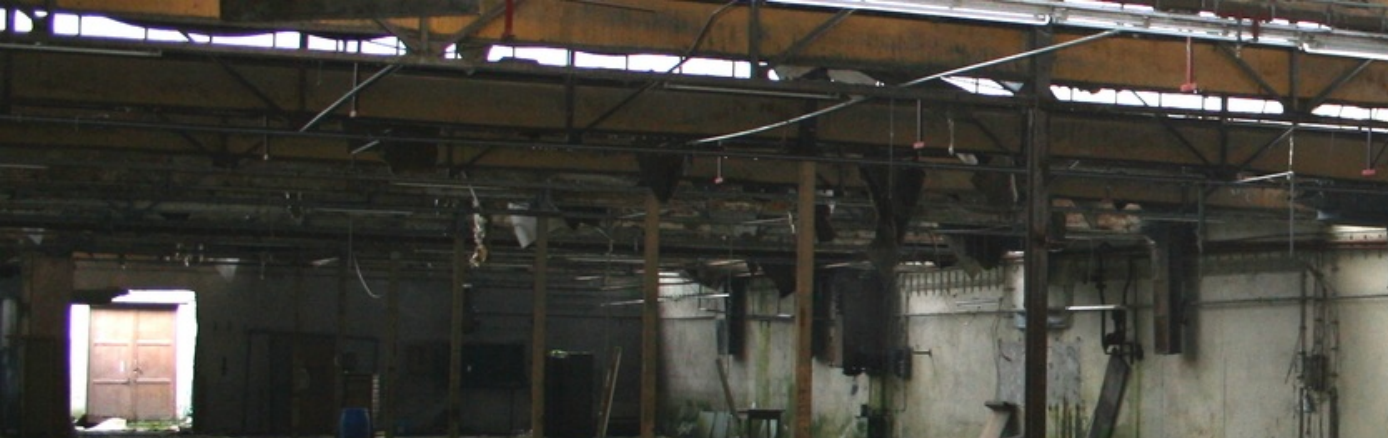

\title{
Industriegeschichte im Bild
}

Authors: Jürgen Meyer-Isenmann

Submitted:

10. October 2015

Published:

10. October 2015

Volume:

Issue:

2

Keywords: Industriegeschichte, Mischtechnik auf Leder und Holz

DOI: $\quad 10.17160 /$ josha.2.6.70

Journal of Science, Humanities and Arts 


\title{
Industriegeschichte im Bild
}

\author{
Arbeiten auf / aus Lederriemen
}

Jürgen Meyer-Isenmann, Juli 2015

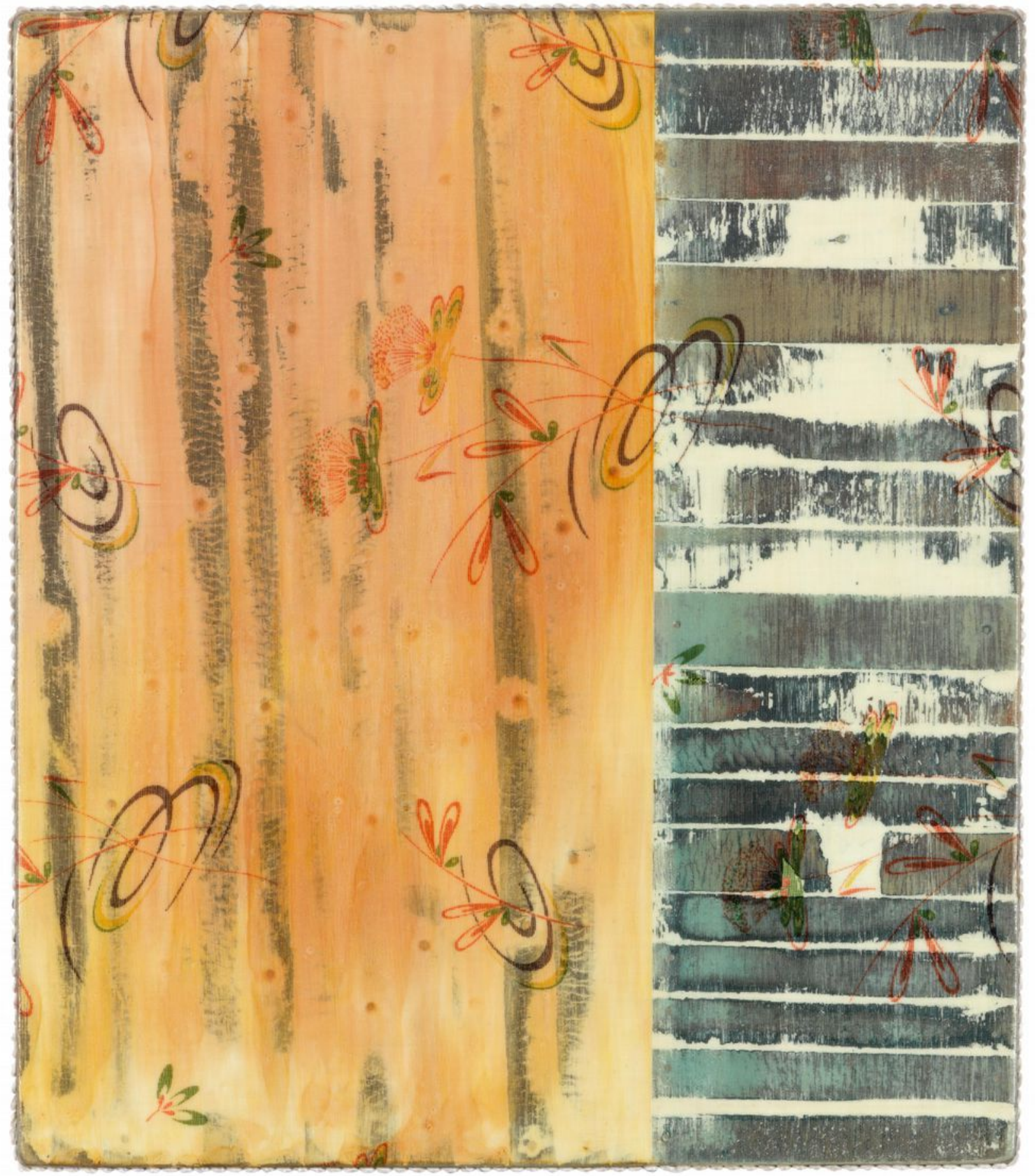

Facette 2

2013, Mischtechnik auf Leder und Holz, 32 × 27,5 cm

Fotografie: Lea Gryze, Berlin 
Ihren Ursprung haben die Arbeiten in den 2001 stillgelegten ELZATextilwerken in Elzach. Während meines Studiums beschäftigte ich mich von 2006 bis 2007 mit den Räumen dieser vom Zerfall bedrohten

Fabrikhallen. Dank eines kunstinteressierten Eigentümers und eines aufgeschlossenen Sparkassendirektors, konnte ich in den Hallen arbeiten und sämtliche Materialien verwenden. Dadurch erhielt ich die Chance sehr außergewöhnliche Bilder zu erarbeiten, mit einer einzigartigen Vielfalt an Materialien. Alte Türen, Textilien, Schränke und vieles mehr wurde zu Bildern verarbeitet. Die Industriegeschichte immer vor Augen, genoss ich die Atmosphäre der zerfallenden Räume und gebrauchten Gegenstände.

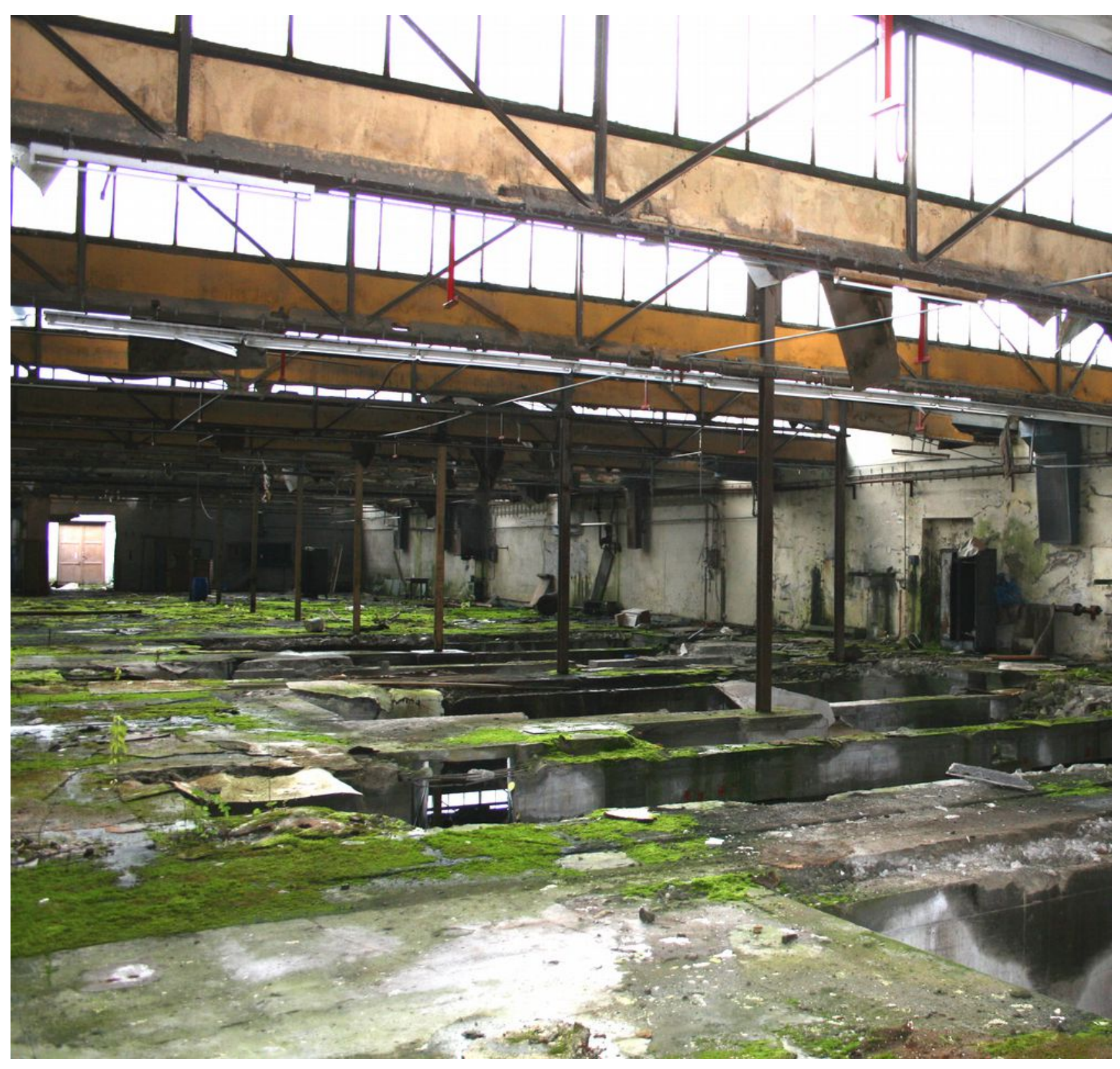


Nachdem 2007 die Ergebnisse des Aufenthalts in einer Ausstellung durch die Stadt Waldkirch gezeigt wurden, entwickelte sich im Anschluss das gefundene und gesammelte Leder als großes Thema meiner Arbeit. Lederriemen, zwei bis drei Zentimeter breit, wenige Millimeter dick und mehrere Meter lang.

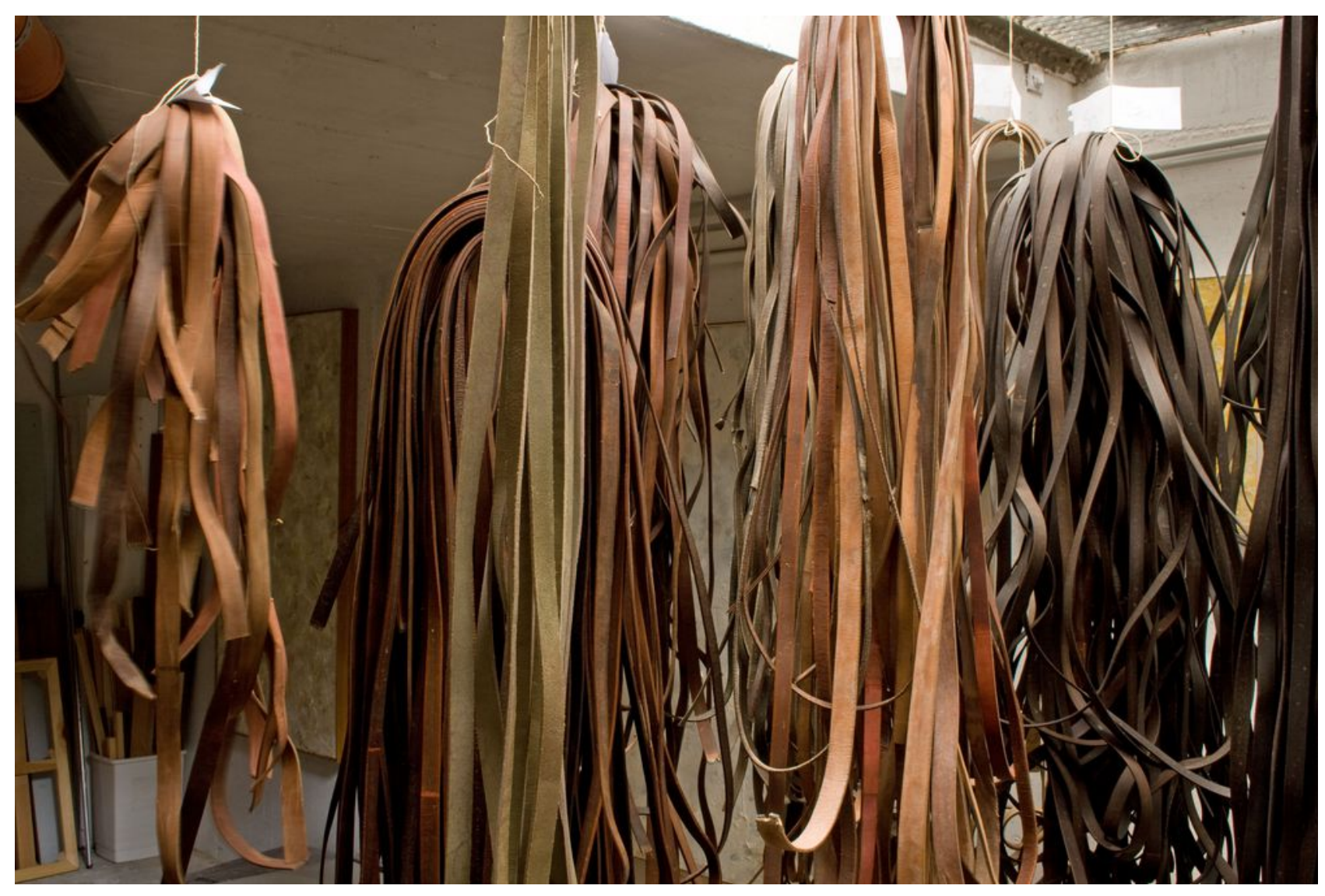

gereinigte Lederriemen

Fotografie: Jürgen Meyer-Isenmann

Bis 2009 entstanden einige Arbeiten, bis eine endgültige Form die Lederriemen zu verwenden gefunden war. Nahtlos aneinandergereiht, ähnlich einem Parkettboden, ergaben sich plane Flächen, die ich weiter gestalten konnte. Mit Ölfarbe bestrichen und nach dem Trocknen geschliffen, entstanden in intermittierenden und langwierigen Arbeitsprozessen subtile Bilder. Folgend entschloss ich mich das restliche Material nach dem gleichen Prinzip zu verwenden. So entstand von 2009 bis 2014 eine Serie von insgesamt 21 Bildern verschiedenster Größe.

Knapp 1000 Meter Leder wurden verarbeitet und damit ein Stück Industriegeschichte in Bildern festgehalten. 
Das außergewöhnliche an dieser Serie ist ihre Abgeschlossenheit, das Material ist aufgebraucht und wird in dieser Form heute nicht mehr hergestellt. Im Nachhinein weiß ich, dass mir diese Abgeschlossenheit besonders wichtig ist. Eine Gruppe von Bildern zusammenzufassen und abzuschließen bedeutet mir die Möglichkeit zu Neuem.

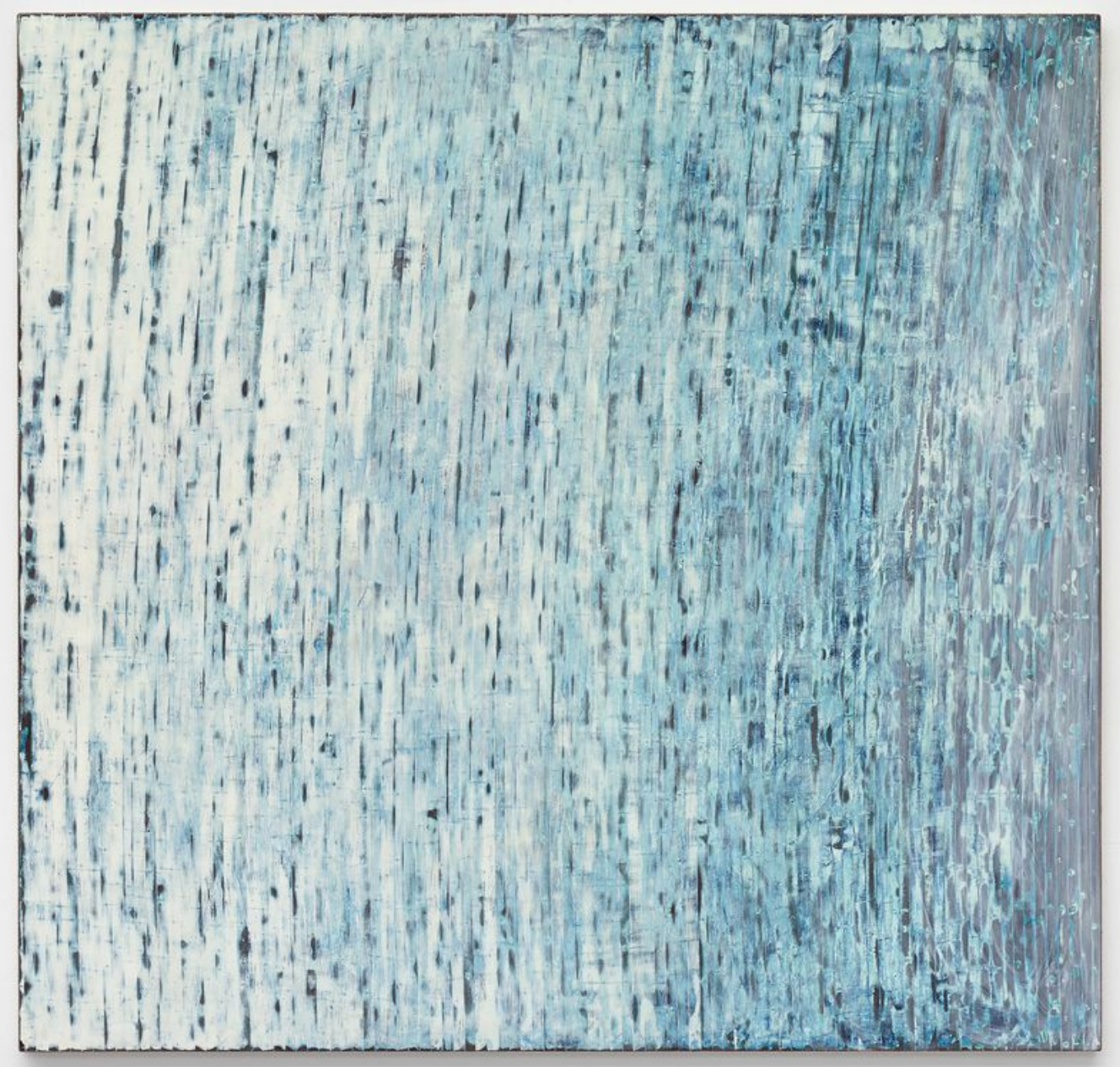




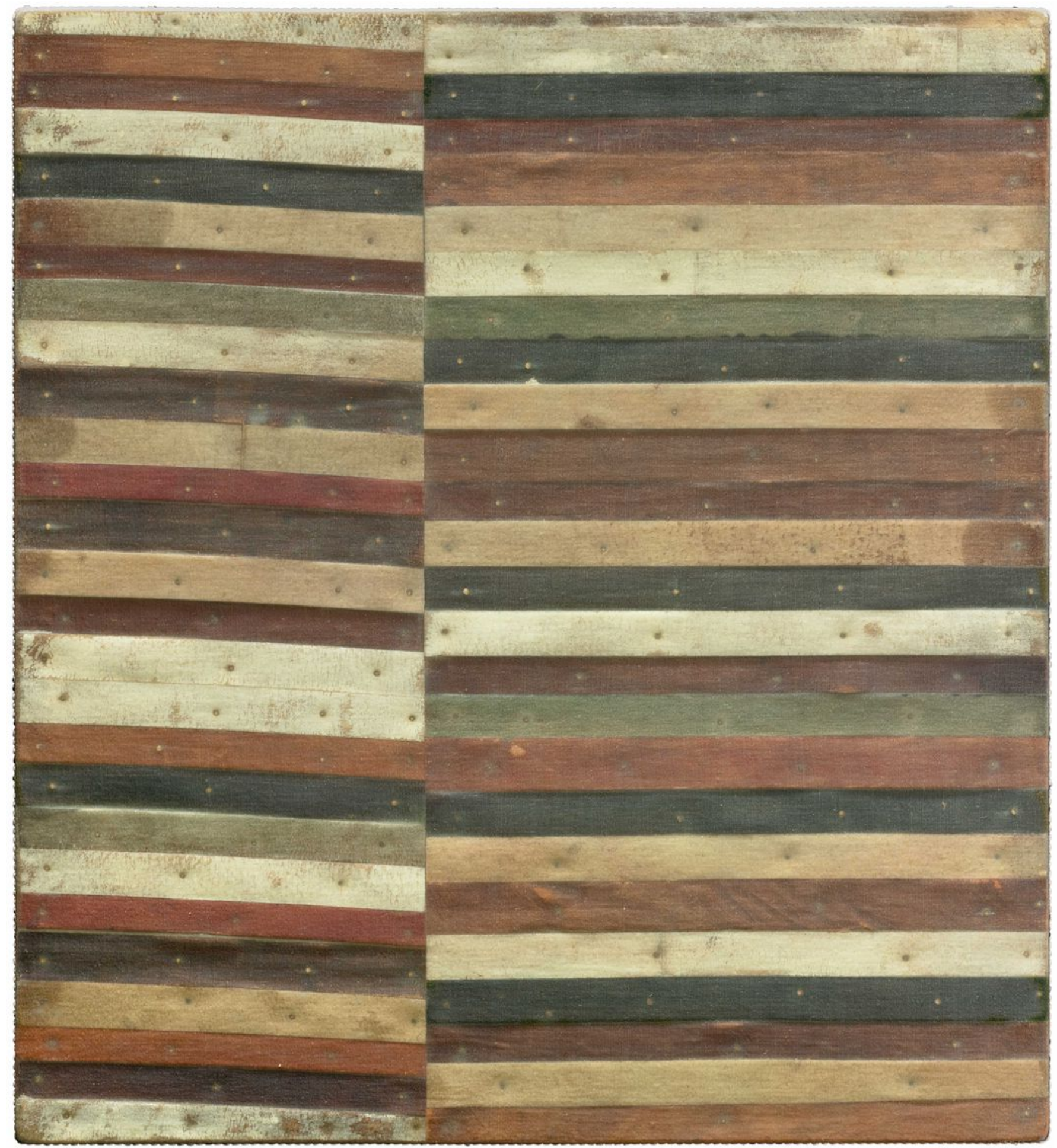

Facette

2010, Mischtechnik auf Leder und Holz, 53,5 x 48,5 cm

Fotografie: Bernhard Strauss, Freiburg

Ähnlich entstand auch eine 7 Meter lange Vitrine, die einen über mehrere Jahre entstandenen 20teiligen Bilderzyklus beinhaltet. Dazu lernte ich an den Lederbildern, dass gute Bildformen Zeit brauchen. Gut insofern, dass sie ihre Wirkung nicht verlieren und uns ein Leben lang beschäftigen. 

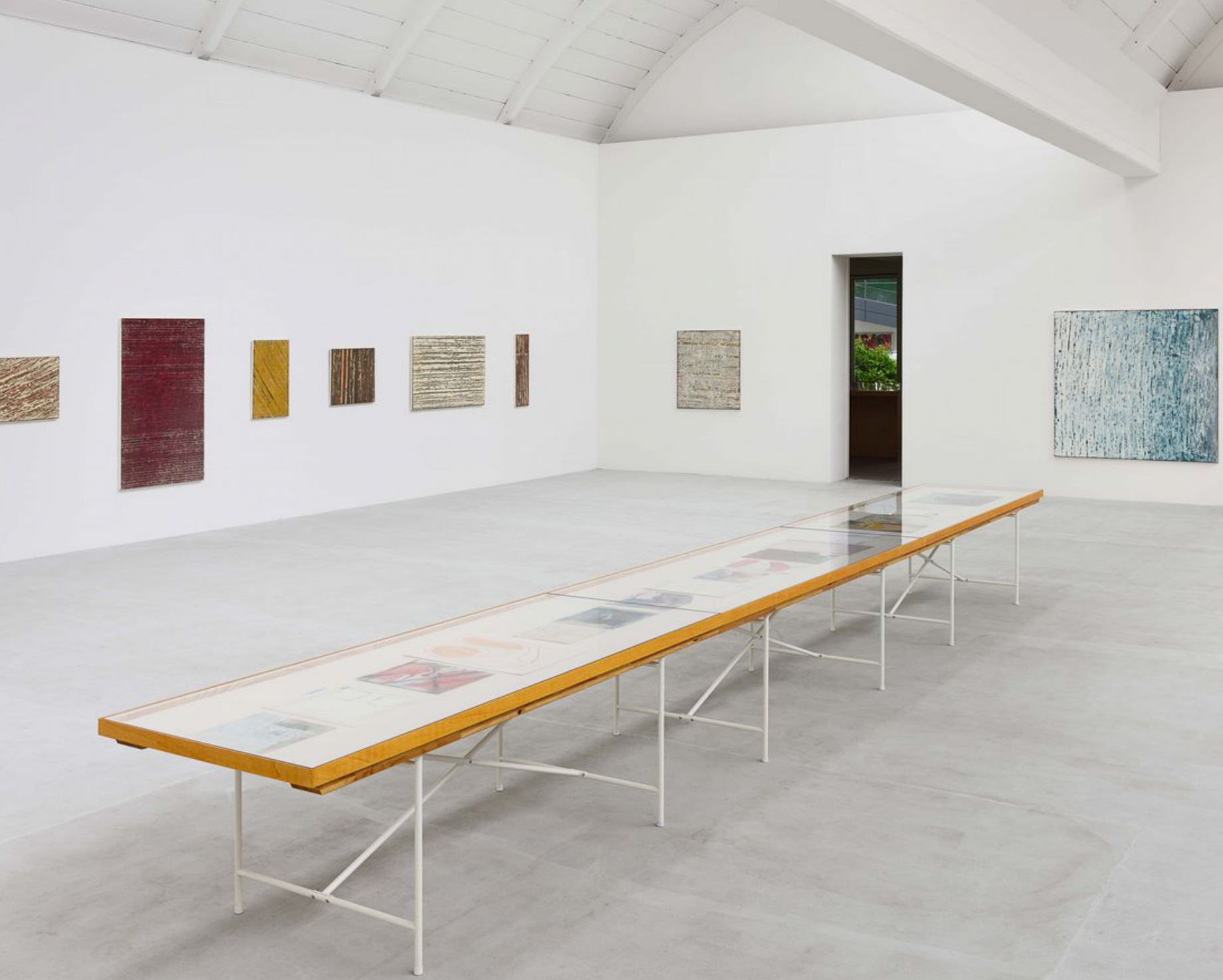

Morat Institut für Kunst und Kunstwissenschaft Freiburg, 2013

Ausstellungsansicht, Lederbilder / Vitrine

Fotografie: Bernhard Strauss, Freiburg

... nächste Ausstellung: 30. Oktober bis 28. November 2015, Galerie Meier Freiburg

weitere Infos unter: www.meyer-isenmann.de 